\title{
Time changes of renal dimensions and variations of glomerular filtration rate in chronic kidney disease patients
}

\author{
Simone Brardi ${ }^{1}$, Gabriele Cevenini ${ }^{2}$ \\ ${ }^{1}$ Hemodialysis Unit, S. Donato Hospital, Arezzo, Italy; \\ 2 Department of Medical Biotechnologies, University of Siena, Italy.
}

\begin{abstract}
Summary Background: The aim of this longitudinal prospective study was to search if even in the absence of total or partial nephrectomy the kidney size can increase as the kidney function improves.

Methods: We randomly enrolled 80 adult patients with various degrees of chronic renal failure but non-dialysis dependent neither totally or partially nephrectomized nor affected by any of the pathological conditions that can increase kidney size. The patients underwent a first examination comprehensive of a blood sample and renal ultrasonography and then were submitted to a therapeutic intervention aimed at removing all nephrotoxic agents to finally be subjected to a last similar medical examination.

Results: The statistical analysis displayed a strong positive correlation between the percentage variation of the renal diameters' average and the time changes of the GFR ( $r$ 0.731; $p<0.01)$ as well as the percentage variation of the GFR and the time changes variations of the right $(r 0.487 ; p<0.01)$ and left cortical kidney thickness $(r 0.519 ; p<0.01)$ and finally a strong negative correlation between the removal of nephrotoxic agents and the percentage variation of the renal diameters' average $(r-0.293 ; p<0.01)$ and the time changes of the GFR $(r-0.429 ; p<0.01)$.

Conclusions: In patients with chronic kidney disease, even in the absence of total or partial nephrectomy, under the stimulus of the removal of any nephrotoxic agents, there may be a limited increase in renal size according to a model that sees them vary according to the changes in GFR.
\end{abstract}

KEY WORDS: Longitudinal prospective study; Renal length variations; GFR time changes; Renal ultrasound; CKD patients.

Submitted 4 October 2019; Accepted 13 November 2019

\section{INTRODUCTION}

The best measure of renal size is the volume which correlates well with the glomerular filtration rate (1).

However, calculation of volume is prone to significant error because three independent measurements are used (2). Therefore, measurement of maximum renal length has become the clinical standard because it is simple, mode accurate and correlates well with renal volume $(3,2)$.

Renal length averages about $11 \mathrm{~cm}$ in adults (3) so that $10-12 \mathrm{~cm}$ is a useful range for normal renal length, the discrepancy in size between the two kidneys is not abnormal provided that the smaller kidney is not less than $37 \%$ of the total renal volume. All measured kidney dimensions of patients with chronic kidney disease (CKD) correlate significantly with kidney function while the correlation with anthropometric parameters which is otherwise present in healthy subjects is lost in patients with $\mathrm{CKD}(4,5)$. More in detail the renal length, as renal failure progresses, significantly decreases (6). Therefore, the sonographic appearance of chronic renal failure consists of a decrease in renal size, thinning of the parenchyma (particularly the cortex) and increased echogenicity of the cortex. When cortical thickness cannot be determined because the medullary pyramids are not visible thinning of the cortex can still be appreciated as thinning of the entire parenchyma defined by a cutoff of $12 \mathrm{~mm}$ (3). The decrease of renal size and cortical thinning are often most noticeable in renovascular disease and hypertensive nephrosclerosis whereas in glomerular disorders particularly diabetic nephropathy the cortical thickness is often preserved even late in the disease (7). Instead, a physiological enlargement is known to occur only in solitary kidneys and during pregnancy while compensatory hypertrophy is seen in adults after nephrectomy (7). Nowadays therefore it is well known that average value of kidney's volume shows a linear decrease with the progression of chronic kidney disease (8) and that kidney volume and its surrogate parameter that is the renal length correlate with GFR $(4,5)$ but we don't know if this process, in the absence of total or partial nephrectomy, may be reversible, because until now it was never evaluated if the kidney length correlated also with the time changes of GFR, as can be recorded in a longitudinal type study. So, in order to investigate a similar renal regrowth in the absence of total or partial nephrectomy, we conducted this longitudinal prospective study.

\section{MATERIALS AND METHODS}

This study was undertaken between June 2015 to September 2019 in the outpatient nephrology clinic of San Donato Hospital in Arezzo. The study population was randomly selected among adult patients with various degrees of chronic renal failure but non-dialysis dependent and affected or not by hypertension but not by diabetes mellitus neither totally or partially nephrectomized and with the exclusion of those with obstructive uropathy, ischemia, pregnancy, polycystic kidney disease, and malignant diseases. After obtaining the respective informed consent from all participating subjects, we enrolled 80 patients ( 35 females and 45 males, with an average age, at the time of enrollment, of $71.5 \pm 11.7$ years) almost all hypertensive (except only four).

The average glomerular filtration rate (GFR) (calculated by the CKD EPI equation) (9) at the time of enrollment, was 
$49.4 \pm 16.8 \mathrm{ml} / \mathrm{min} / 1.73 \mathrm{~m}^{2}$. After the evaluation of medical history including the complete list of medications and every dietetic therapy, we submitted the enrolled patients to an accurate physical examination, comprehensive of the recording of anthropometric parameters (weight and height) and of a measurement of blood pressure that was taken with a mercury sphygmomanometer applied around each patient's non-dominant arm after the patient had rested for 15 minutes in a sitting position and with his/her arm placed at the level of the heart. Two consecutive blood pressure recordings, taken at a 5-minute interval, were averaged to provide clinic systolic and diastolic blood pressure values. Blood samples were taken for serum creatinine and sodium and, where possible, 24-hour urine collection for albuminuria was executed. Finally, a B-mode and Doppler renal ultrasound was performed. All renal ultrasound examinations were carried out by the same nephrol-

\section{Table 1.}

Basic and final characteristics of the enrolled population and percentage changes (mean \pm standard deviation).

\begin{tabular}{|c|c|c|c|}
\hline Parameters & Basal values $F$ & Follow-up values & Delta \\
\hline Age (years) & $71.5 \pm 11.7$ & $73.99 \pm 11.39$ & $3.72 \pm 3.47$ \\
\hline Weight (kg) & $74.3 \pm 11.7$ & $72.4 \pm 13$ & $-2.37 \pm 7.37$ \\
\hline Body mass index (BMI) $\left(\mathrm{kg} / \mathrm{m}^{\wedge} 2\right)$ & $28.3 \pm 4.7$ & $27.6 \pm 4.9$ & $-2.36 \pm 7.4$ \\
\hline Glomerular filtration rate (GFR) (ml/min/1.73 m^2) & $49.4 \pm 17$ & $50.6 \pm 15.2$ & $6.93 \pm 32.4$ \\
\hline 24-hour urinary albumin excretion (gr/24h) & $0.2 \pm 0.75$ & $0.13 \pm 0.6$ & $-7.31 \pm 98.5$ \\
\hline Right kidney diameter in the longitudinal axis (mm) & $101.7 \pm 9.7$ & $101.91 \pm 8.37$ & $0.70 \pm 8.5$ \\
\hline Left kidney diameter in the longitudinal axis (mm) & $103.4 \pm 10.4$ & $103.43 \pm 9.23$ & $0.33 \pm 7$ \\
\hline renal diameters' average in the longitudinal axis (mm) & $102.6 \pm 9.4$ & $102.67 \pm 7.84$ & $0.47 \pm 6.9$ \\
\hline Right kidney Renal resistive index & $0.73 \pm 0.1$ & $0.72 \pm 0.07$ & $-0.54 \pm 10$ \\
\hline Left kidney renal resistive index & $0.73 \pm 0.1$ & $0.72 \pm 0.08$ & $-0.68 \pm 9.7$ \\
\hline Blood serum natrium (mmol/L) & $142 \pm 2.2$ & $141.82 \pm 2.30$ & $-0.16 \pm 2.2$ \\
\hline Systolic blood pressure (mmHg) & $122.6 \pm 17.3$ & $117.79 \pm 16.9$ & $-2.6 \pm 16.7$ \\
\hline Diastolic blood pressure (mmHg) & $75.9 \pm 11.7$ & $72.38 \pm 12.68$ & $-3.4 \pm 19.3$ \\
\hline Mean arterial pressure (mmHg) & $91.4 \pm 11$ & $87.51 \pm 11.81$ & $-3.3 \pm 16.4$ \\
\hline
\end{tabular}

\section{Table 2.}

Rates of patients having a preserved kidney cortical thickness $(\geq 1.2 \mathrm{~cm})$ or a slightly reduced kidney cortical thickness $(>1<1.2 \mathrm{~cm})$ or a reduced kidney cortical thickness $(<1 \mathrm{~cm})$.

\begin{tabular}{|lccc}
\hline Number of patients & Basal values & Follow-up values & Delta \\
\hline Number of patients having a preserved right kidney cortical thickness & $28(35 \%)$ & $30(37.5 \%)$ & 7.14 \\
\hline Number of patients having a preserved left kidney cortical thickness & $25(31.25 \%)$ & $28(35 \%)$ & 12.00 \\
\hline Number of patients having a slightly reduced right kidney cortical thickness & $19(23.75 \%)$ & $22(27.5 \%)$ & 15.79 \\
\hline Number of patients having a slightly reduced left kidney cortical thickness & $23(28.75 \%)$ & $24(30 \%)$ & 4.35 \\
\hline Number of patients having a reduced right kidney cortical thickness & $33(41.25 \%)$ & $28(35 \%)$ & -15.15 \\
\hline Number of patients having a reduced left kidney cortical thickness & $32(40 \%)$ & $28(35 \%)$ & -12.50 \\
\hline
\end{tabular}

Table 3.

Rates of patients taking different drugs or being on a specific diet.

\begin{tabular}{|lccc|}
\hline Number of patients (percentage of the total) & Basal values & Follow-up values & Delta \\
\hline Number of patients having hypo protein diet & $24(30 \%)$ & $57(71.25 \%)$ & 137.5 \\
\hline Number of patients having hyposodic diet & $24(30 \%)$ & $62(77.5 \%)$ & 158.33 \\
\hline Number of patients taking ACE inhibitors or angiotensin receptor blockers & $58(72.5 \%)$ & $52(65 \%)$ & -10.34 \\
\hline Number of patients taking diuretics & $36(45 \%)$ & $22(27.5 \%)$ & -38.89 \\
\hline Number of patients taking nephrotoxic drugs & $26(32.5 \%)$ & $1(1.25 \%)$ & -96.15 \\
\hline
\end{tabular}

ogist experienced in ultrasound examination using the same ultrasound device that was a Logiq S7 (GE Medical Systems Italy S.P.A. Milan, Italy) sonographic system equipped with 3 to $5 \mathrm{Mhz}$ transducers. Any dimensional parameter of each kidney (i.e. length or else diameter in the longitudinal axis and parenchymal thickness) was registered as the average of two single measurements.

The diameter in the longitudinal axis was assessed in a section visually estimated to represent the largest diameter. The cortical thickness was registered in the portion closer to the upper pole and the lower pole of the same kidney (4). Doppler signals were obtained from the interlobar arteries from the upper, middle and lower third of both kidneys and resistive index was calculated as the average of 6 measurements (3 from each of the 2 kidneys) taken for each patient. The Doppler angle was chosen as close to $0^{\circ}$ as possible and special care was taken not to compress the kidney and not to have the patient performing Valsalva maneuver because both of them can increase the renal resistive index value. Then the patients were submitted to a full therapeutic and dietetic intervention to ameliorate the renal impairment by a wide range of actions such as removal of nephrotoxic drugs (i.e. the hydrochlorothiazide diuretics, non-steroidal anti-inflammatory drugs, etc.) as well as any variation of drug therapy in order to reduce proteinuria and improve blood pressure control avoiding both too low blood pressure values and those that are too high (10). Eventually, when it was indicated, it was introduced an hypoproteic, hyposodic as well as hypocaloric diet (11). After on average of $2.5( \pm 2.2)$ year interval the same patients were submitted to a second and last medical examination (to evaluate the outcome of the therapeutic and/or dietetic actions undertaken) which was conducted in the same way as the first one. Sample descriptive statistics were calculated, including mean and standard deviation for quantitative variables, and frequency counts and percentages for qualitative variables (Tables 1-3). A Pearson correlation analysis was performed between quantitative variables, particularly between the time changes of GFR and the average of left/right renal diameters. The linear correlation coefficient, r, was computed and its statistical significance was evaluated at a minimum level of $95 \%(\mathrm{p}<0.05)$. All the statistical computations were executed using the SPSS package, version 10.

\section{RESULTS}

In order to investigate if the dimensional parameters of the kidney correlate or not with the variations of the GFR, we compared the percentage variation between basal and final examination (Table 4), or Delta, of each variable with the percentage variation of the renal diameters' average in the longitudinal axis and the time changes of GFR (Figure 1).

The statistical analysis so displayed first a strong positive correlation between the Delta of the average renal diameter in the longitudinal axis and time changes of GFR ( $\mathrm{r} 0.731 ; \mathrm{p}<$ 0.01 ) and then an equally strong positive correlation between the same Delta of GFR and 
Table 4.

Statistically significant correlations.

\begin{tabular}{|c|c|c|c|}
\hline Variables & & $\begin{array}{l}\text { Delta of the renal } \\
\text { diameters' average }\end{array}$ & $\begin{array}{c}\text { Delta } \\
\text { of the GFR }\end{array}$ \\
\hline \multirow[t]{3}{*}{ Delta of the renal diameters' average } & $r$ & 1 & 0.731 \\
\hline & $p$ & i & $<0.001$ \\
\hline & $\mathrm{N}$ & l & 80 \\
\hline \multirow[t]{3}{*}{ Delta of the GFR } & $r$ & 0.731 & 1 \\
\hline & $p$ & $<0.001$ & 1 \\
\hline & N & 80 & l \\
\hline \multirow[t]{3}{*}{ Delta of the age at the moment of the examination } & r & -0.360 & -0.304 \\
\hline & $p$ & 0.001 & 0.006 \\
\hline & N & 80 & 80 \\
\hline \multirow[t]{3}{*}{ Delta of the systolic blood pressure } & $r$ & 0.238 & 0.370 \\
\hline & $p$ & 0.035 & 0.001 \\
\hline & $\mathrm{N}$ & 79 & 79 \\
\hline \multirow[t]{3}{*}{ Delta of the mean arterial pressure } & $r$ & 0.190 & 0.289 \\
\hline & $p$ & 0.093 & 0.01 \\
\hline & N & 79 & 79 \\
\hline \multirow[t]{3}{*}{ Delta of the right kidney diameters } & $r$ & 0.907 & 0.661 \\
\hline & $p$ & $<0.001$ & $<0.001$ \\
\hline & N & 80 & 80 \\
\hline \multirow[t]{3}{*}{ Delta of the left kidney diameters } & r & 0.866 & 0.637 \\
\hline & $p$ & $<0.001$ & $<0.001$ \\
\hline & N & 80 & 80 \\
\hline \multirow[t]{3}{*}{ Delta of the right kidney cortical thickness } & r & 0.506 & 0.487 \\
\hline & $p$ & $<0.001$ & $<0.001$ \\
\hline & N & 80 & 80 \\
\hline \multirow[t]{3}{*}{ Delta of the left kidney cortical thickness } & r & 0.535 & 0.519 \\
\hline & $p$ & $<0.001$ & $<0.001$ \\
\hline & N & 80 & 80 \\
\hline \multirow[t]{3}{*}{ Delta of the number of patients having a hyposodic diet } & r & 0.068 & 0.250 \\
\hline & $p$ & 0.546 & 0.025 \\
\hline & N & 80 & 80 \\
\hline \multirow[t]{3}{*}{ Delta of the number of patients taking diuretics } & $r$ & -0.262 & -0.226 \\
\hline & $p$ & 0.019 & 0.044 \\
\hline & N & 80 & 80 \\
\hline \multirow[t]{3}{*}{ Delta of the number of patients taking nephrotoxic drugs } & $r$ & -0.293 & -0.429 \\
\hline & $p$ & 0.008 & $<0.001$ \\
\hline & N & 80 & 80 \\
\hline
\end{tabular}

\section{Figure 1.}

A graphical representation of the correlation that was found between the percentage variation of the average of the renal diameters and the time changes of the GFR with respect to the values at the time of the enrollment.

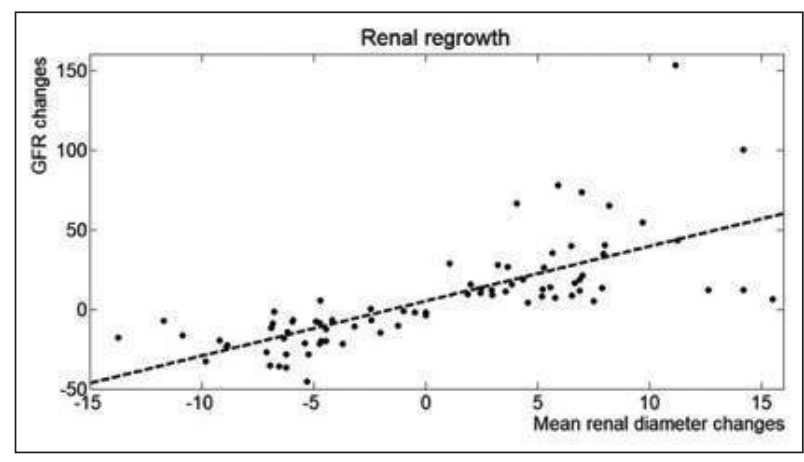

the percentage variations of the right ( 0.487 ; $p<0.01$ ) and left (r 0.519; p <0.01) kidney cortical thickness. As obvious a similarly strong correlation was found between time changes of right ( $\mathrm{r} 0.661 ; \mathrm{p}<0.01)$ and left ( $\mathrm{r} 0.637 ; \mathrm{p}<0.01$ ) kidney diameters in the longitudinal axis and the time changes of the GFR. Instead, no statistical correlation was found between time changes of GFR and percentage variations of right or left renal resistive indices as well as body mass index and proteinuria. About the percentage variations of the blood pressure parameters we found a strong positive correlation only between the time changes of systolic blood pressure and the percentage variation of GFR ( $\mathrm{r} 0.370 ; \mathrm{p}<0.01$ ) and a weaker positive correlation between the same Delta of systolic blood pressure and the time changes of renal diameters' average in the longitudinal axis ( $\mathrm{r} 0.238$; $\mathrm{p}<0.05)$, moreover a strong positive correlation was found between the Delta of the mean arterial pressure and the Delta of GFR ( $\mathrm{r} 0.289$; $\mathrm{p}<$ 0.01). A weak negative correlation was found between the strong reduction of the diuretics usage and the Delta of the renal diameters' average in the longitudinal axis ( $r-0.262$; $\mathrm{p}<0.05)$ or the Delta of GFR ( $\mathrm{r}-0.226$; $\mathrm{p}<0.05)$ while a stronger negative correlation was found between the almost complete removal of any nephrotoxic drugs and the Delta of the renal diameters' average in the longitudinal axis ( $\mathrm{r}-0.293$; $\mathrm{p}<0.01$ ) or the Delta of GFR ( $\mathrm{r}-0.429$; $\mathrm{p}<$ 0.01 ). A weak positive correlation was found between the introduction of a low-salt diet and the time changes of GFR (r 0.250; p < 0.05) but no statistical correlation was found between the Delta of the renal diameters' average in the longitudinal axis or the Delta of GFR and the variations of serum sodium, the introduction of an low protein diet or the usage of ACE inhibitors or angiotensin receptor blockers. Finally a strong negative correlation was found between the time changes of the age at the moment of the examination and the Delta of the renal diameters' average in the longitudinal axis $(\mathrm{r}-0.360 ; \mathrm{p}<0.01)$ and the time changes of GFR ( $r-0.304 ; p<0.01)$.

\section{Discussion}

In this longitudinal prospective observational type study comparing the average of left and right renal diameters and the time changes of GFR we found a strong positive correlation between the two ones as well as a similar strong positive correlation between the time changes of the GFR and percentage variations of the right and left kidney cortical thickness. All the above means that the renal sizes may increase or decrease synchronously with the time changes of GFR even in absence of the classic conditions that promote the compensatory renal hypertrophy (i.e. mainly radical or partial nephrectomy) as well as of any other condition that may increase the renal dimensions such as mainly the diabetes mellitus. Now it is well known that after unilateral radical or partial nephrectomy a compensatory renal growth, promoting significant restoration of lost renal function, is observed in the remnant kidney. This compensatory renal growth following nephrectomy is predominantly due to a renal hypertrophy (i.e. an increase in cell size) dominated by tubular cells (since mainly proximal tubules show increase of length, diameter and volume) and produces a GFR increase so can be termed physiologic compensatory renal hypertrophy in contrast to the so-called pathologic renal hypertrophy that, typical of diabetic nephropathy, it is dominated by a glomerular and podocyte hypertrophy and can lead to further nephron damage, interstitial fibrosis and ultimately end-stage kidney disease (12). Lastly, it is known that compensatory kidney growth was not only found to occur 
after renal mass reduction by partial nephrectomy but also after partial renal parenchymal damage induced by nephrotoxins $(13,14)$. Now in this study we exercised a decise action aimed at removing all nephrotoxic pharmacological agents and the most common ones that we eliminated at the time of the enrollment were thiazide diuretics with the use of which apoptosis has been observed in the distal tubular cells, just those that dominate the physiological renal hypertrophy (15). Therefore, it seems probable that the removal of nephrotoxic agents done in this study may be the action that mostly induced compensatory kidney growth. About the strong positive correlation that we found between time changes of systolic blood pressure and percentage variations of the GFR and the weaker positive correlation found between Delta of the systolic blood pressure and time changes of renal diameters' average in longitudinal axis, we suppose that when in $\mathrm{CKD}$, aorta is stiffed, a decrease of systolic blood pressure can limit the renal perfusion that, in this condition, is mostly dependent by stroke volume, causing a decrease of GFR, reversible with the restoration of systolic blood pressure (10). In fact at the time of enrollment, as part of our action to improve kidney function, we accurately corrected not only the high systolic blood pressure values but also those excessively reduced (i.e. next or less than 100 $\mathrm{mmHg}$ ) which in the population enrolled were relatively frequent. To the characteristics of the population enrolled in terms of systolic blood pressure, can be probably due the correlation that was found between the reduction of diuretics usage (which can significantly contribute to reducing the systolic blood pressure) and the time changes of renal diameters'average in the longitudinal axis or the Delta of the GFR.

Lastly, as known, another parameter that correlates with kidney length is age: in adults there is a gradual reduction in renal length that becomes more precipitous after age 50 years and that is due entirely to loss of parenchyma. (4, 3, 16). This likely can explain the strong negative correlation that was found between percentage variation of age at the moment of the examination and Delta of the average of renal diameters in the longitudinal axis and time changes of GFR. It is our opinion therefore that, in the chronic kidney disease patients, also in the absence of total or partial nephrectomy as well as of any other condition that may increase the renal dimensions such as mainly diabetes mellitus, under the stimulus of the removal of any agent that may hamper renal function (such as, mainly, nephrotoxic drugs or excessively reduced values of systolic blood pressure), can be found a limited increase of the renal sizes according to a model that sees the same renal dimensions reduce or increase depending on changes in the GFR. Moreover, in the chronic kidney disease patients, repeated accurate ultrasound measurements of the kidney sizes correlate significantly with the variations of kidney function and therefore can be used as a tool to confirm the trend of renal function.

\section{AUTHORS' CONTRIBUTIONS}

Each author declares to have fully participated in the drafting of the work and assumes all public responsibility for the content.

\section{REFERENCES}

1. Troell S, Berg U, Johansson B, et al. Comparison between renal parenchymal sono graphic volume, renal parenchymal urographic area, glomerular filtration rate and renal plasma flow in children. Scan J Urol Nephrol. 1988; 22:207.

2. Emamian SA, Nielsen MB, Pedersen JT. Intraobserver and interobser variations in sonographic measurements of kidney size in adult volunteers. Acta Radiol. 1995; 36:399.

3. Emamian Sa, Nielsen MB, Pedersen JF, et al. Kidney dimensions at sonography: correlation with age, sex, and habitus in 665 adult volunteers. AJR 1993; 160:83.

4. Jovanovic D, Gasic B, Pavlovic S, Naumovic R. Correlation of kidney size with kidney function and anthropometric parameters in healthy subjects and patients with chronic kidney diseases. Ren Fail. 2013; 35:896-900.

5. Sanusi AA, Argundade FA, Famirewa OC, et al. Relationship of ultrasonographically determined kidney volume with measured GFR, calculated creatinine clearance and other parameters in chronic kidney disease (CKD). Nephrol Dial transplant. 2009; 24:1690-1694.

6. Mazzotta L, Sateschi LM, Carlini A, Antonelli A. Comparison of renal ultrasonographic and functional biometry in healthy patients and in patients with chronic renal failure. Arch Ital Urol Androl. 2002; 74:206-9

7, O’Neill W. C. Sonography of the normal kidney. In: O’Neill W. C. Atlas of renal ultrasonography. WB Saunders Company, Philadelphia, USA: 2001; pp. 10-19.

8. Zubovic SV, Kristic S, Pasic IS. Relationship between ultrasonographically determined kidney volume and progression of chronic kidney disease. Med Glas (Zenica) 2016; 13:90-94.

9. Levey AS, Stevens LA, et al. A new equation to estimate glomerular filtration rate. Ann Intern Med. 2009; 150:604-612.

10. Brardi S, Cevenini G. Low systolic blood pressure values, renal resistive index measurement and glomerular filtration rate in a nondialysis dependent chronic kidney disease population. Arch Ital Urol Androl. 2019; 90:288-292.

11. Di Iorio B, De Santo NG, Anastasio P, et al. The GiordanoGiovannetti diet. J Nephrol. 2013; 26(Suppl. 22):143-152.

12. Rojas-Canales DM, Li JY, Makuei L, Gleadle JM. Compensatory renal hypertrophy following nephrectomy: When and how? Nephrology (Carlton). 2019; 24:1225-1232.

13. Cleper R. Mechanisms of compensatory renal growth. Pediatric Endocrinology reviews (PER) 2012; 10:152-63.

14. Wesson LG. compensatory growth and other growth responses of the kidney. Nephron. 1989; 51:149-184

15. Reungjui S, Pratipanawatr T, Johnson RJ, Nakagawa T. Do thiazides worsen metabolic syndrome and renal disease? The pivotal roles for hyperuricemia and hypokalemia. Curr Opin Nephrol Hypertens. 2008; 17:470-476.

16. Miletic D, Fuckar Z, Sustic A, et al. Sonographic measurement of absolute and relative renal length in adults. J Clin Ultrasound. 1998; 26:185.

\section{Correspondence}

Simone Brardi, MD (Corresponding Author) sibrardi@gmail.com

Hemodialysis Unit, S. Donato Hospital, Arezzo (Italy)

Gabriele Cevenini, MD

Department of Medical Biotechnologies, University of Siena (Italy) 